\title{
تطبيقات الواقع المعزز في مجالات الهندسة المعمارية بين الفرص والتحديات
}

\section{أمنية محمد شوقي أحمد '. ، حسن محمد كامل ‘، فاطمة مصطفى النخيلي '.}

الملخص:

يوضح هذا البحث العوامل المؤثرة على إستخدام تقنيات الواقع المعزز في مجالات الهندسة المعمارية

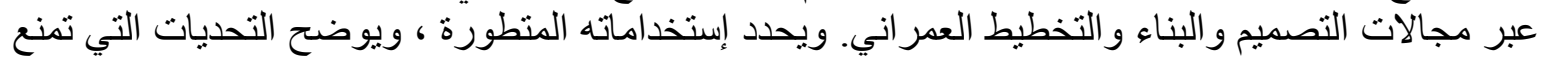

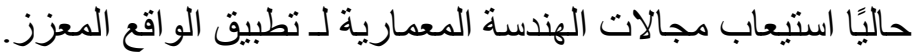

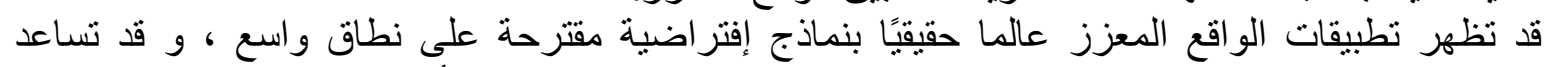

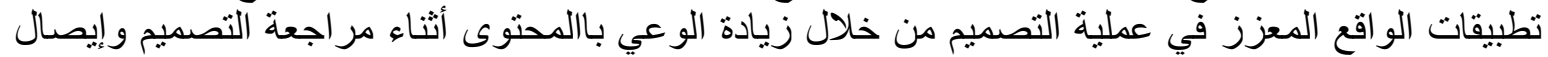
الفكر المعماري، قد تقوم تطبيقات AR بتحديد المواقع الجغرافية لبيانات BIM مباتشرة في موقع البناء

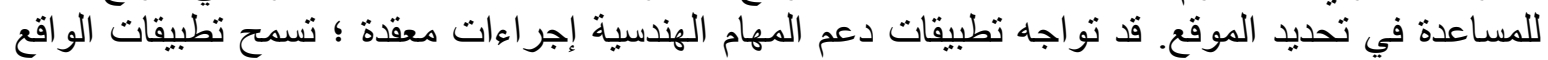
المعزز في الوقت الحقيقي بوضع العلامات الجغر افية للعناصر مباشرة في الموقع. قد يمتد إستخدام تطبيق

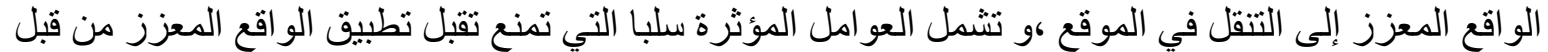

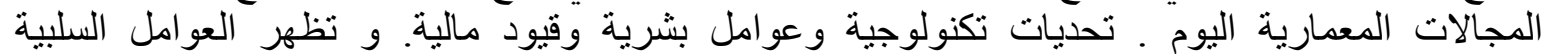

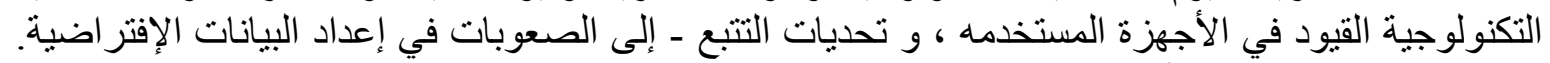

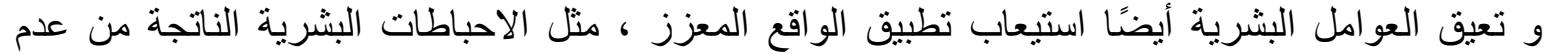

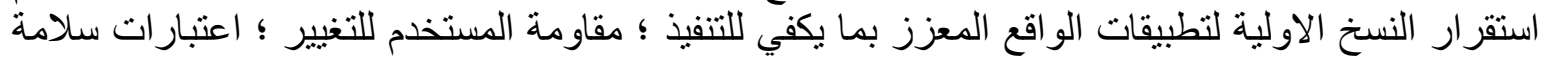

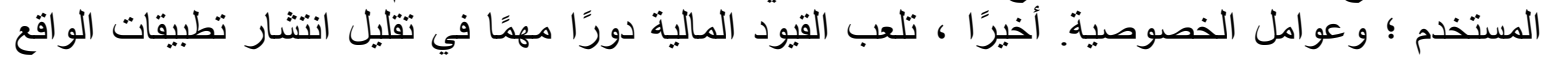

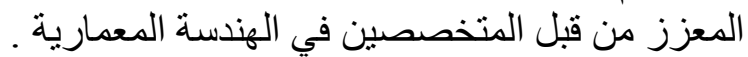

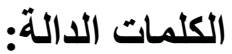

الو اقع المعزز ، الواقع الإفتراضي ، التحديات التكنولوجية ، العوامل البشرية ، القيود المالية.

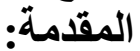

إن مزايا الواقع المعزز في الهندسة المعمارية هائلة. الواقع المعزز هو أي نظام يمذج العالم الحقيقي

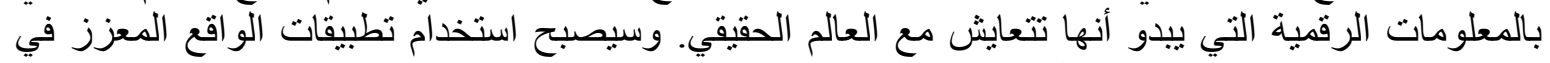

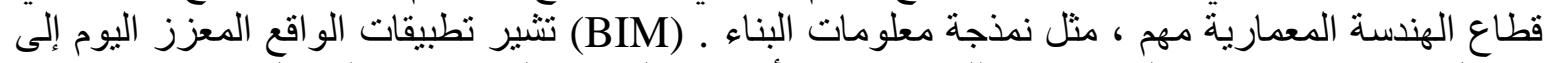

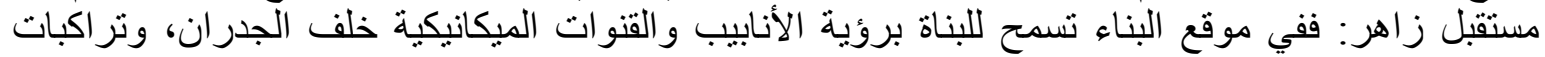

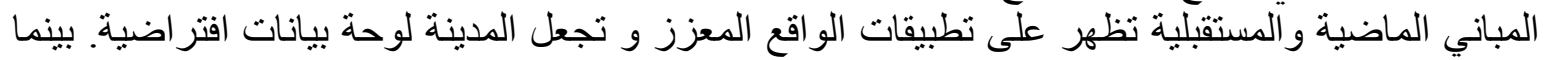

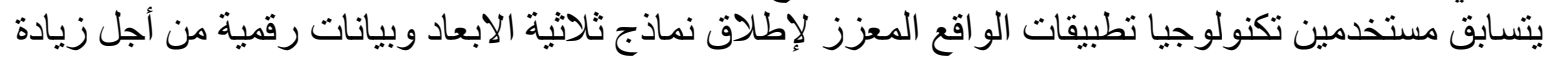

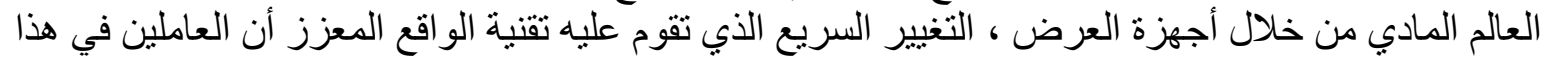

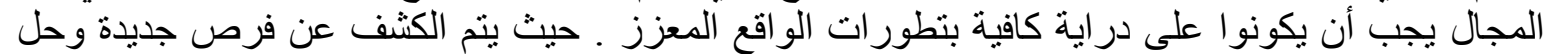

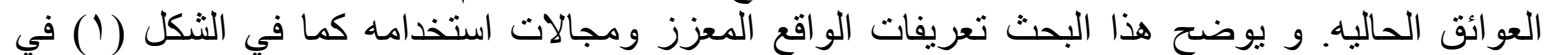

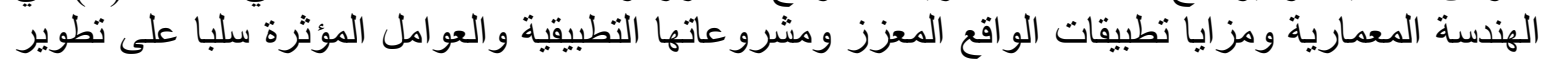

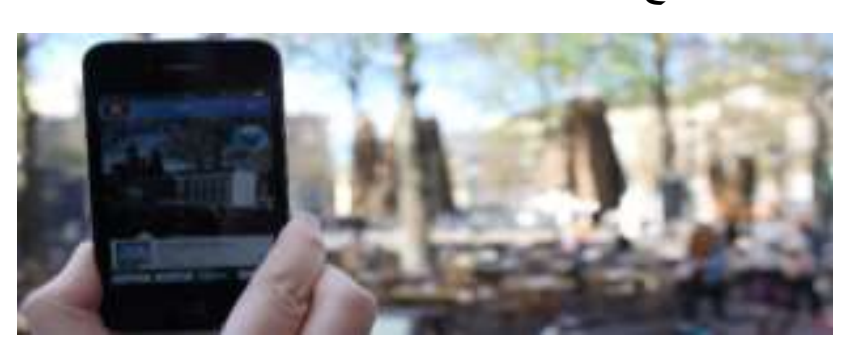

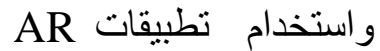

شكل ( 1 ) نطبيق UAR Underground ، يعرض المباني الماضية والمستقبلية في Den Haag )

(1) (1) مدرس مساعد بقسم الهندسة المعمارية بأكاديمية الثروق.

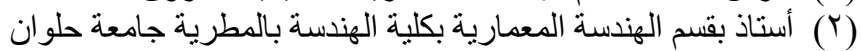

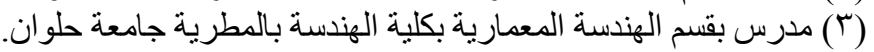




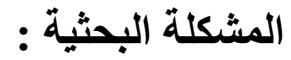

معوقات تطور الواقع المعزز والمعادة العوامل المؤثرة سلبا على مدي الاستفادة القصوى منه في مجالات الهندسة

1 - تحدد العو امل المؤثرة سلبا على الاستفادة القصوى منه .

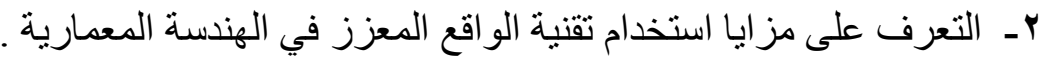

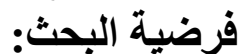

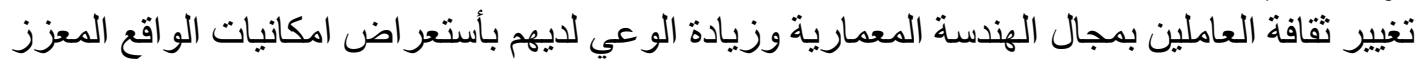

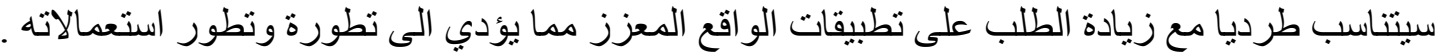

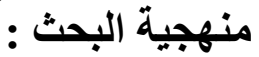

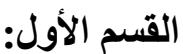

1 - تعريف تقنية الواقع الافتر اضي و المعزز و الفرق بينهم.

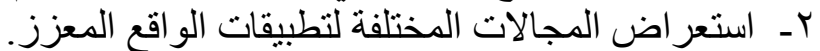

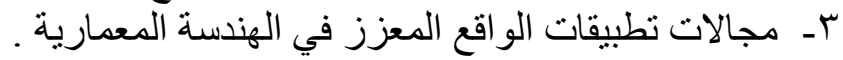

القسم الثاني : الت

ا ـ ـ عرض استخدام تقنية الواقع المعزز ومشروعاتها التطبيقية في مجال التصميم المعماري وأعمال البناء .

r- مجالات تطبيقات الواقع المعزز في الهندسة المعمارية ومشرو عاتها التطبيقية .

القسم الثالث : القالث

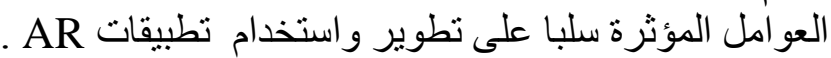

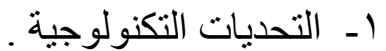

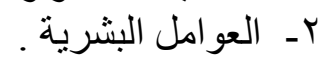

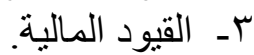

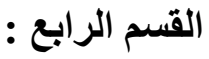

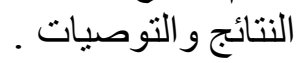

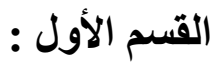

اولا : تعريف تقنية الواقع الافتراضي والمعزز والفرق بينهم:

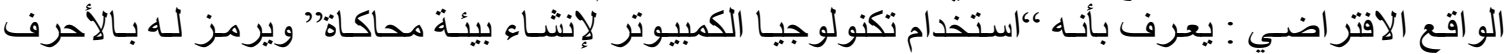

"VR".

الو اقع المعزز (AR) : ع عبارة عن دمج المحتوى الذي تم إنشاؤه بو اسطة الكمبيوتر في العالم الحقيقي.

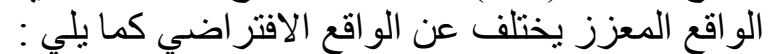

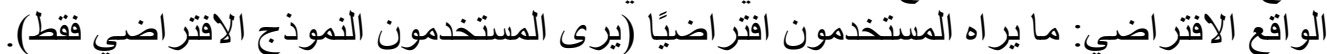

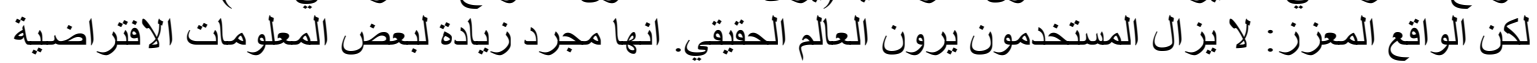

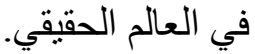

ثانيا: المجالات المختلفة لتطبيقات الواقع المعزز :

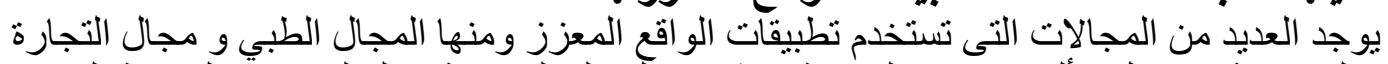

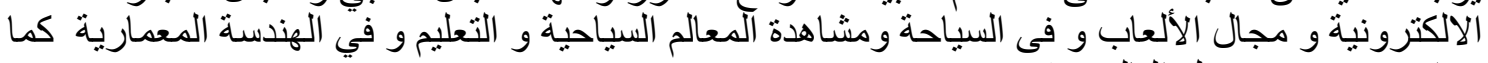
هو الاكترونة في جدول التالي (1) (1) .

(1)https://www.youtube.com/watch?v=uqYAzklhORE 


\begin{tabular}{|c|c|}
\hline & المجالات المختلفة المعزز \\
\hline 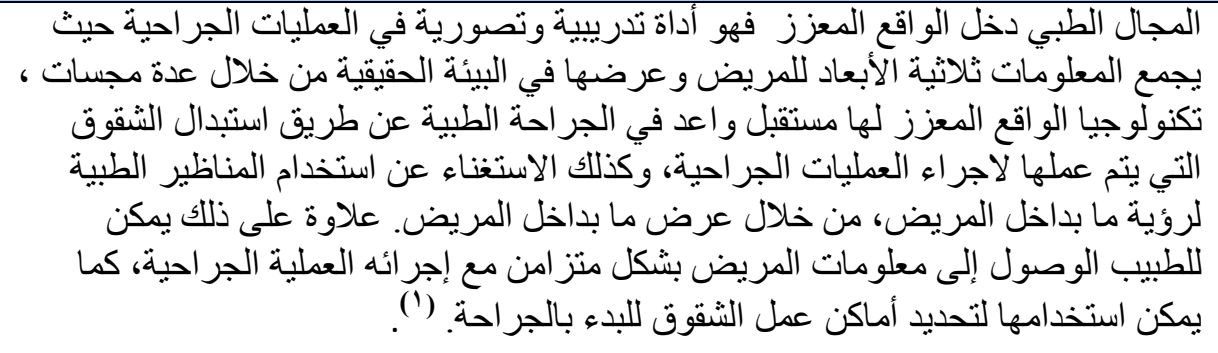 & الواقع المعزز في \\
\hline 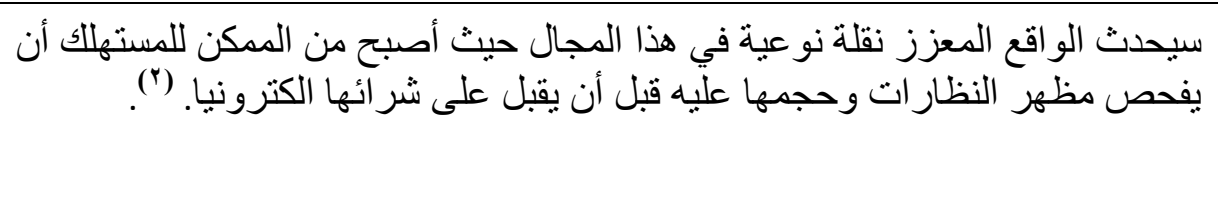 & الواقع المعزز في التجارة في \\
\hline 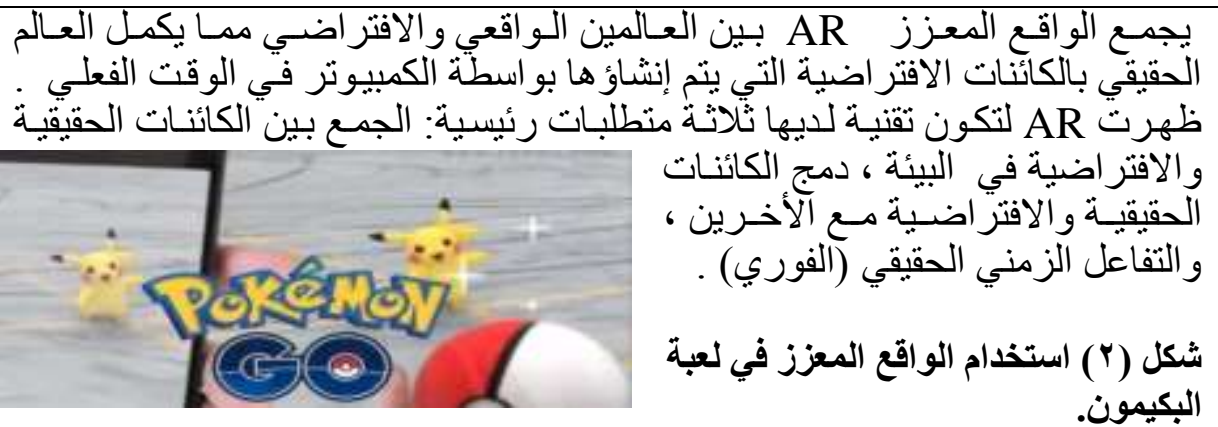 & \\
\hline 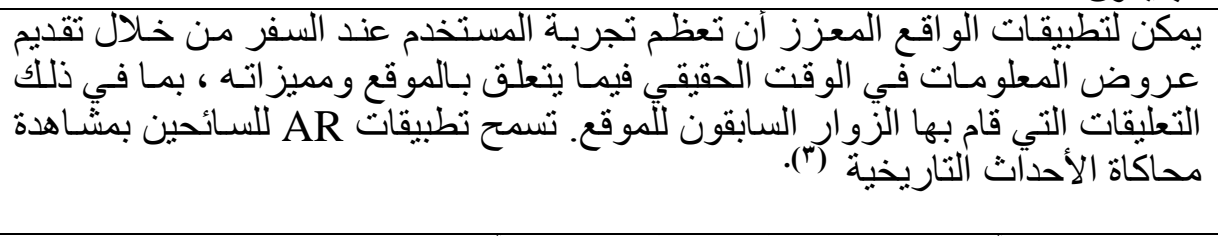 & 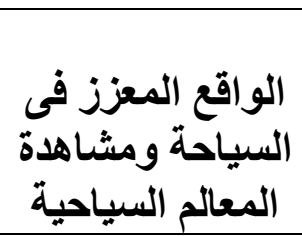 \\
\hline 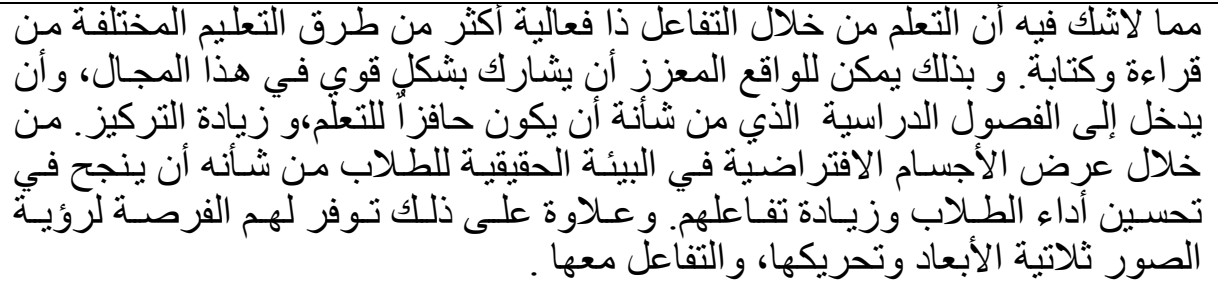 & المعزز في التقبل الواقع \\
\hline 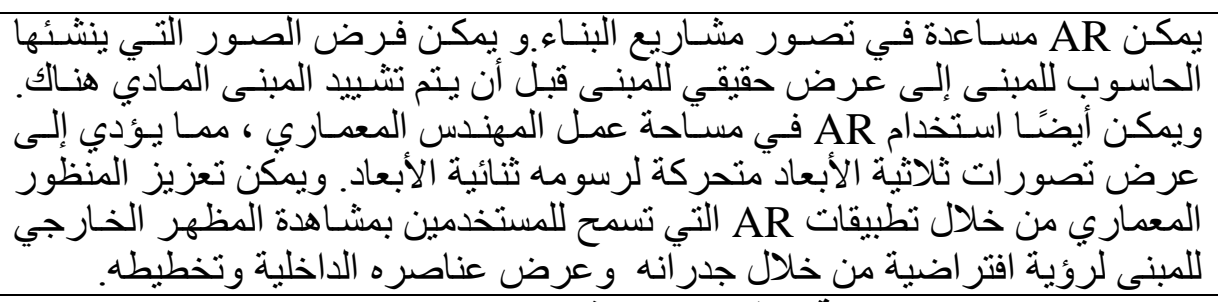 & الوزاقع المعزز في \\
\hline
\end{tabular}
جدول ( 1) يوضح المجالات المختلفة لتطبيقات الواقع المعزز من عمل الباحث.

(1) http://computer.howstuffworks.com/augmented-reality.htm' .

( $\left.{ }^{(}\right) \mathrm{http}: / / \mathrm{www} \cdot \mathrm{t}$-immersion.com/augmented-reality/future-vision، .

( ) http://www.pocket-lint.com/news/108891-augmented-reality-travel-tourism-apps, 2013. 
ثالثا: مجالات تطبيقات الواقع المعزز في الهندسة المعمارية :

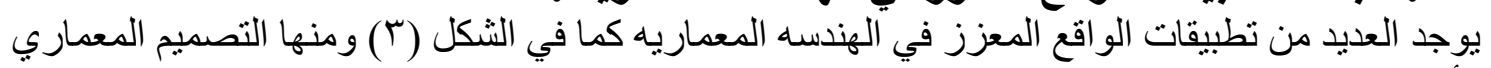

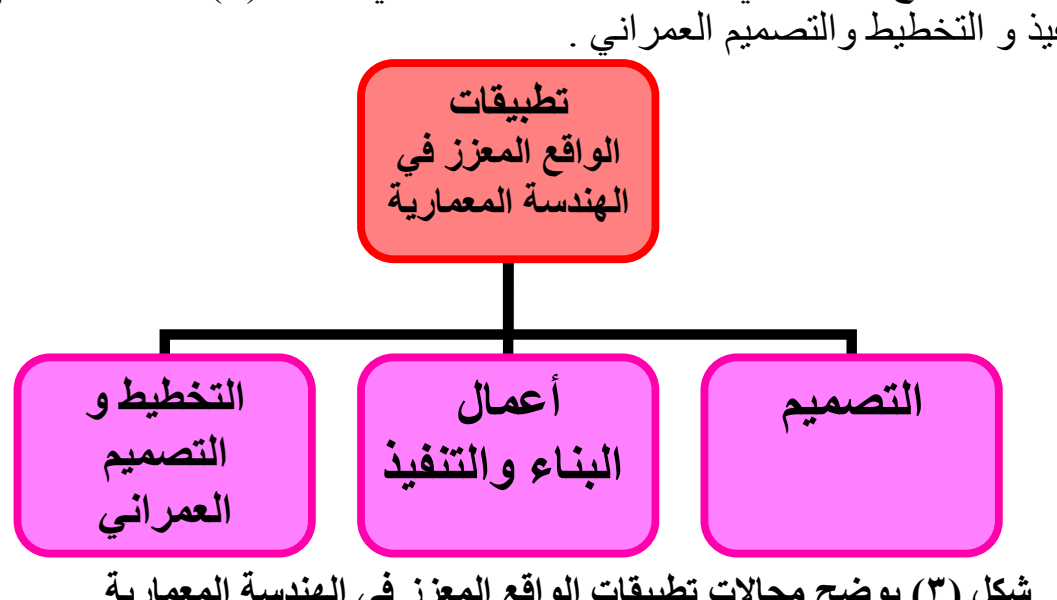

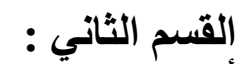

أولا : عرض أنتئ أتخدام تقتية الواقع المعزز ومشروعاتها التطبيقية في مجال التصميم المعماري و

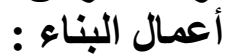

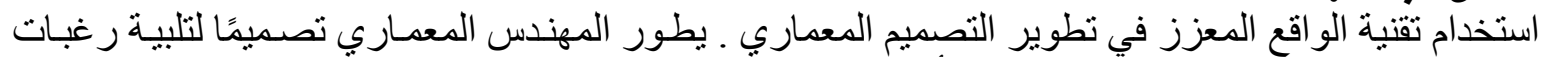

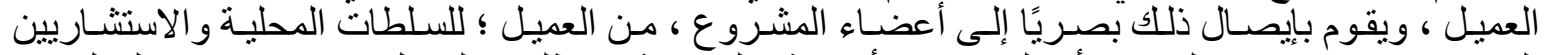

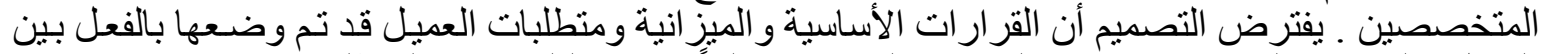

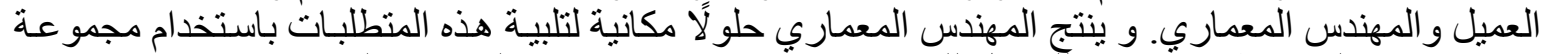

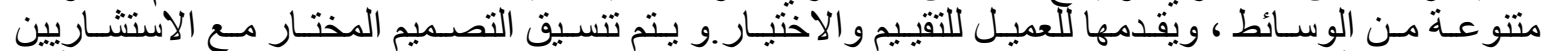

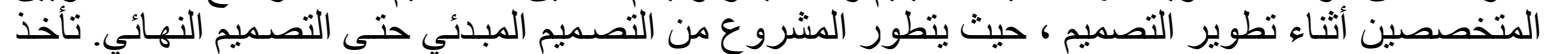

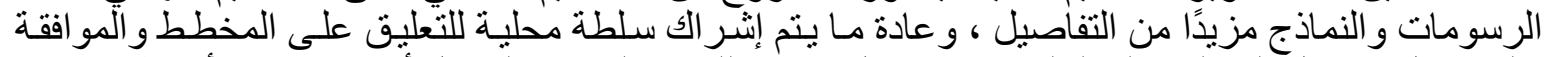

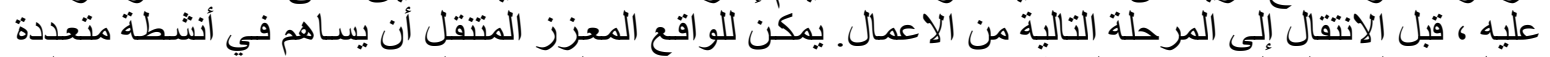

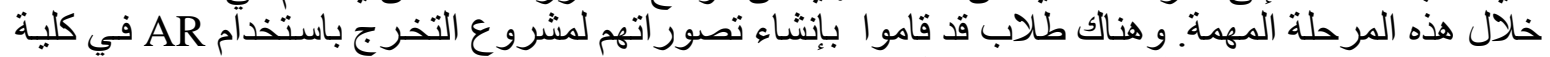

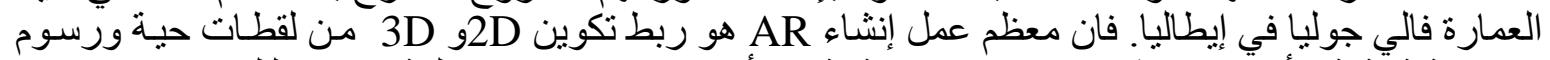

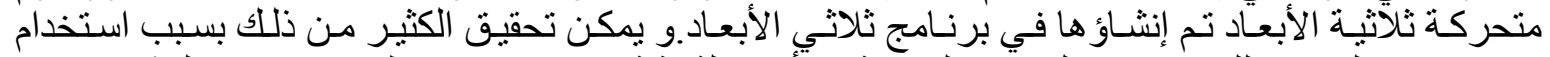

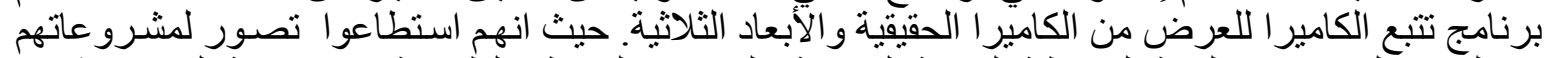

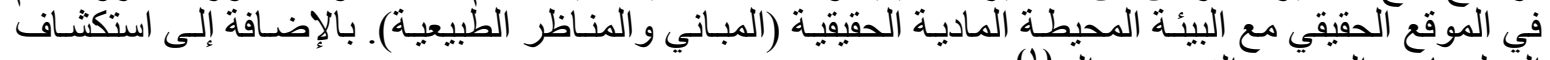

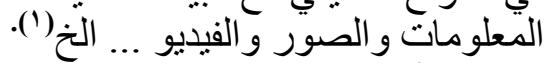
عند بدء أعمال البناء سيكون المهندس المعماري وفريق المشروع من الاستشاريين قد أعدوا بالفعل رسومات

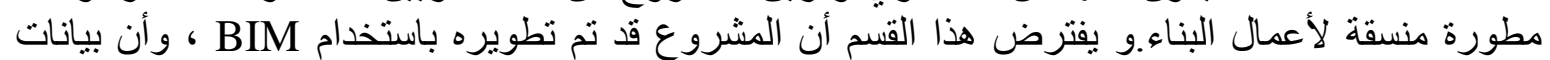

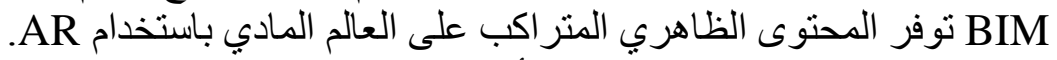

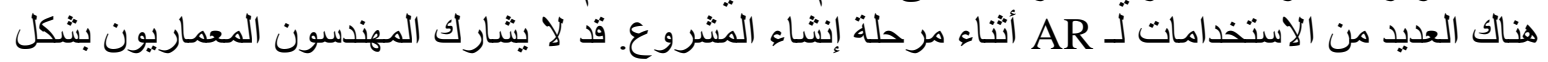

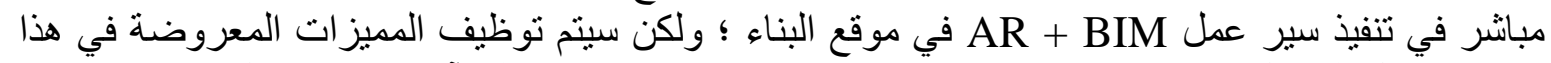

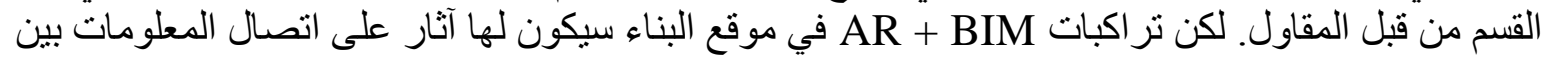

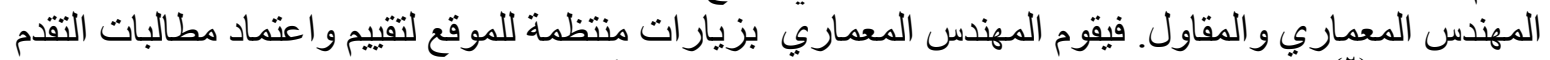

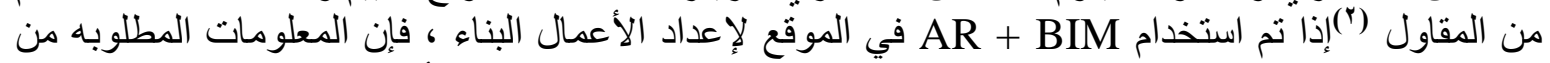

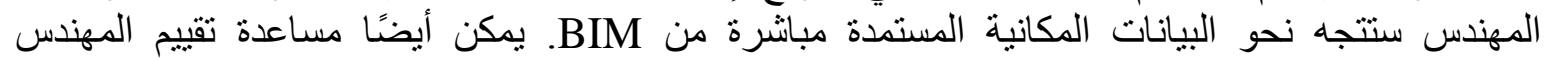
المعماري للتقام في الموقع باستخدام AR.

( ${ }^{\top}$ )http://www.blender3darchitect.com/2009/07/augmented-reality-and-architecturalvisualization/ 2010

(2) Sugar \& Holmes, Royal Australian Institute of Architects and Practice Services, You and Your Architect. Practice Services resource, April 1999. 
ثانيا : مجالات تطبيقات الواقع المعزز في الهندسة المعمارية ومشروعاتها التطبيقية :

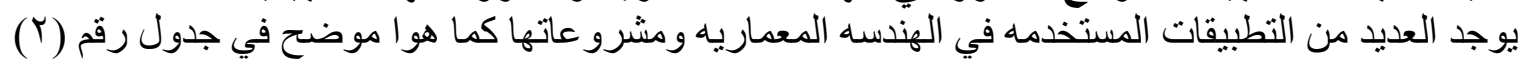
الذي يوضح المجال المستخدم فيه التطبيق وايضا صور الطنئ لاستخدام التطبيق.

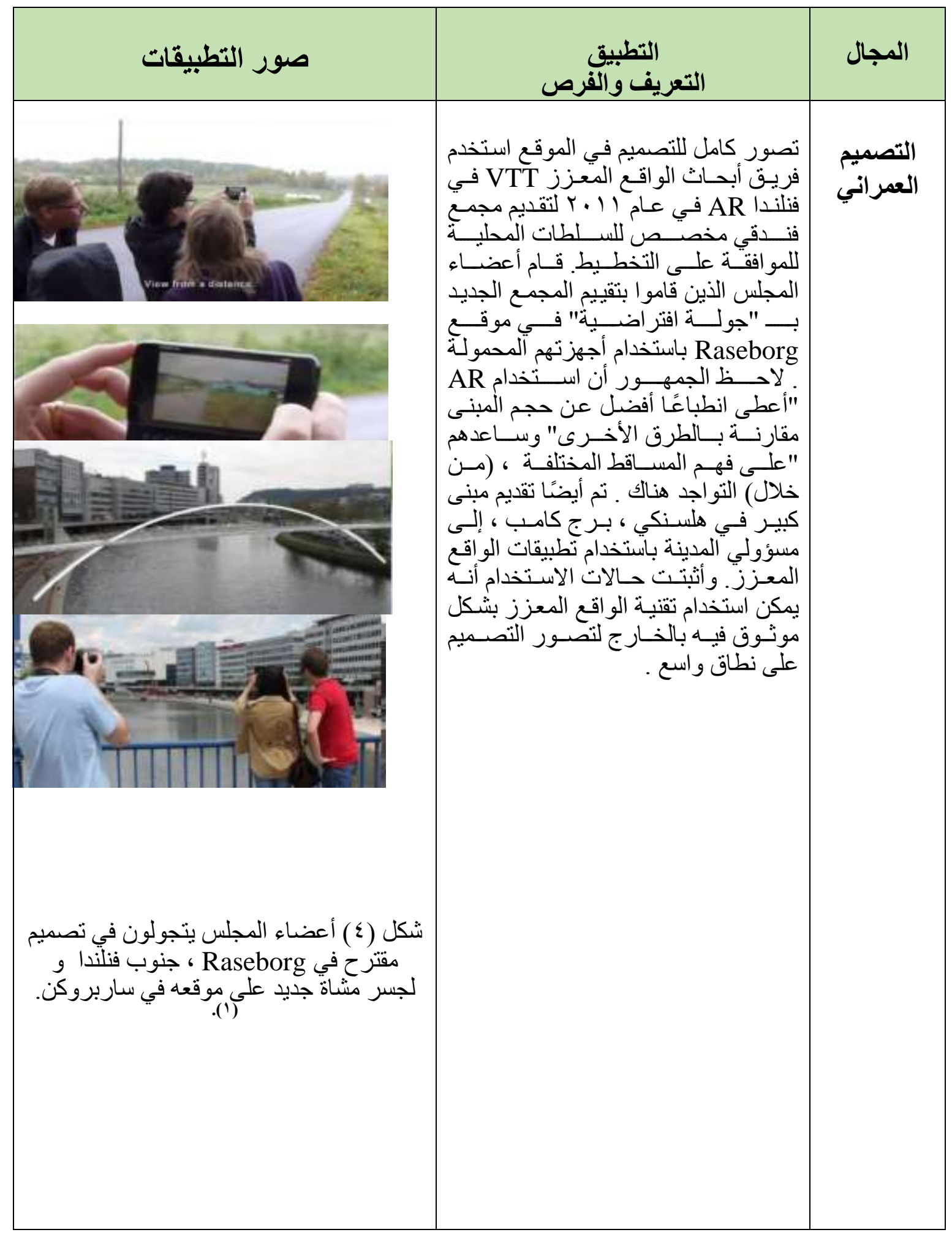

(1) https://www.youtube.com/watch?v=uqYAzklhORE 


\begin{tabular}{|c|c|c|}
\hline صور التطبيقات & التعريف والفرص & المجال \\
\hline 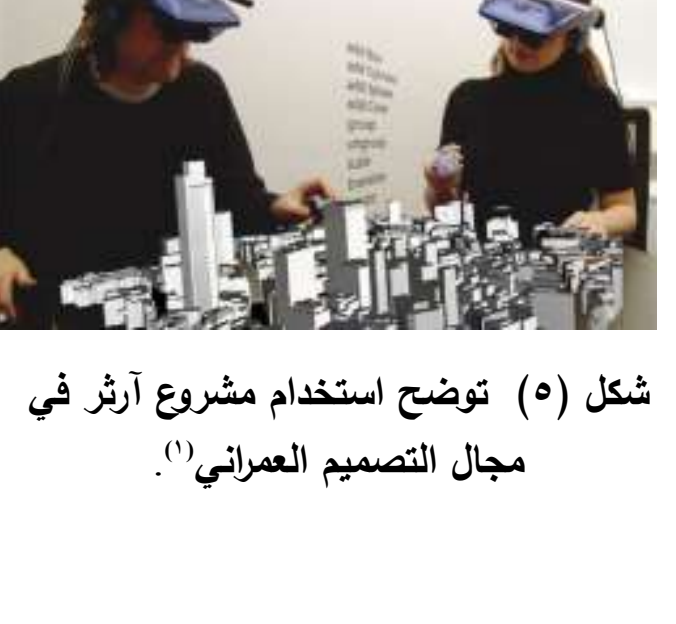 & 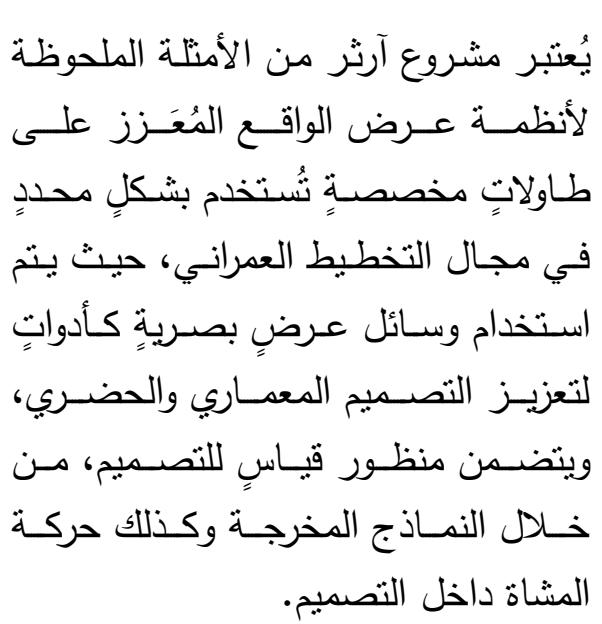 & التخمراني \\
\hline 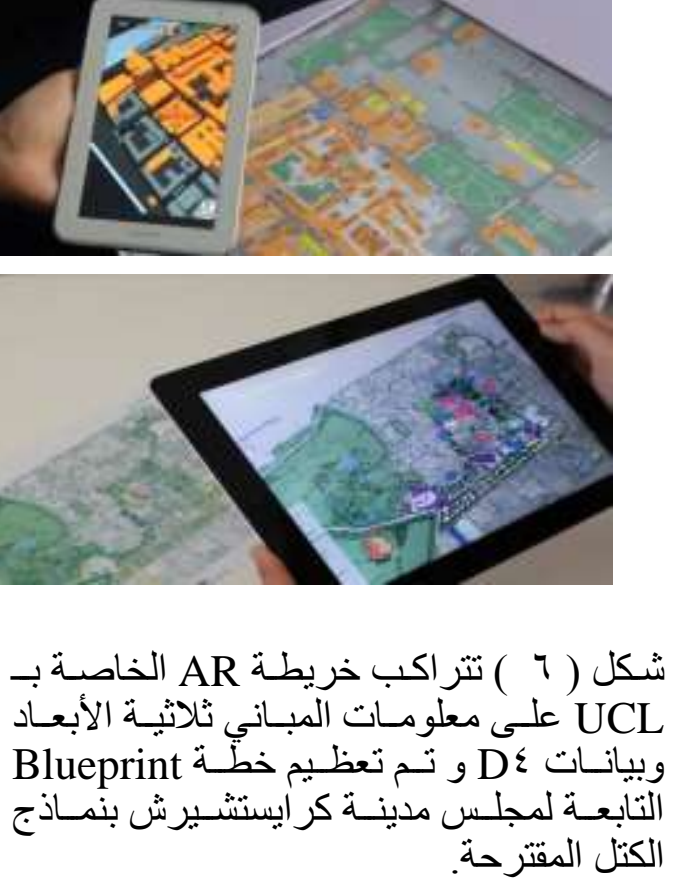 & 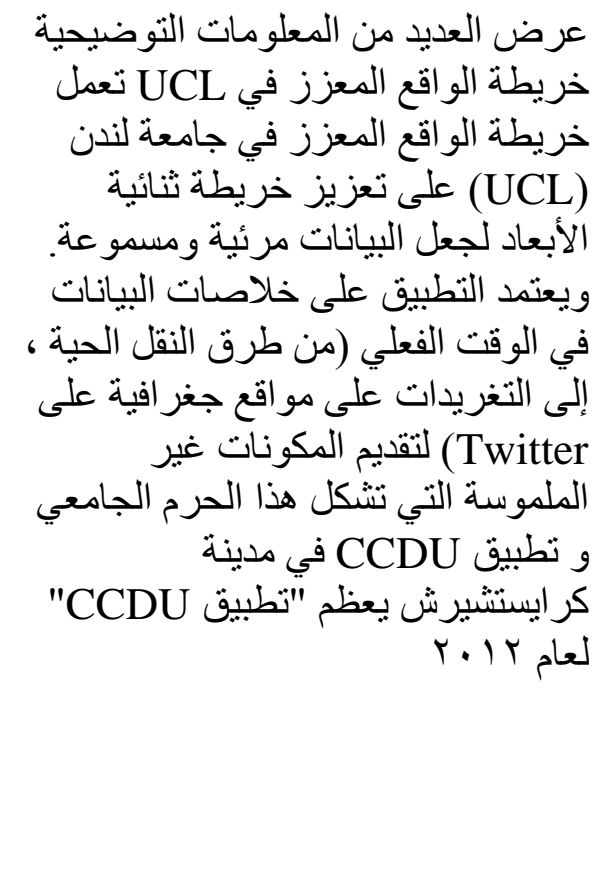 & التمر انيطي \\
\hline
\end{tabular}

(1) Wang X. Augmented Reality in Architecture and Design: Potentials and Challenges for Application. International Journal of Architectural Computing [Internet]. 2009 Jun [cited 2018 Oct 6];7(2):309-26. Available from 


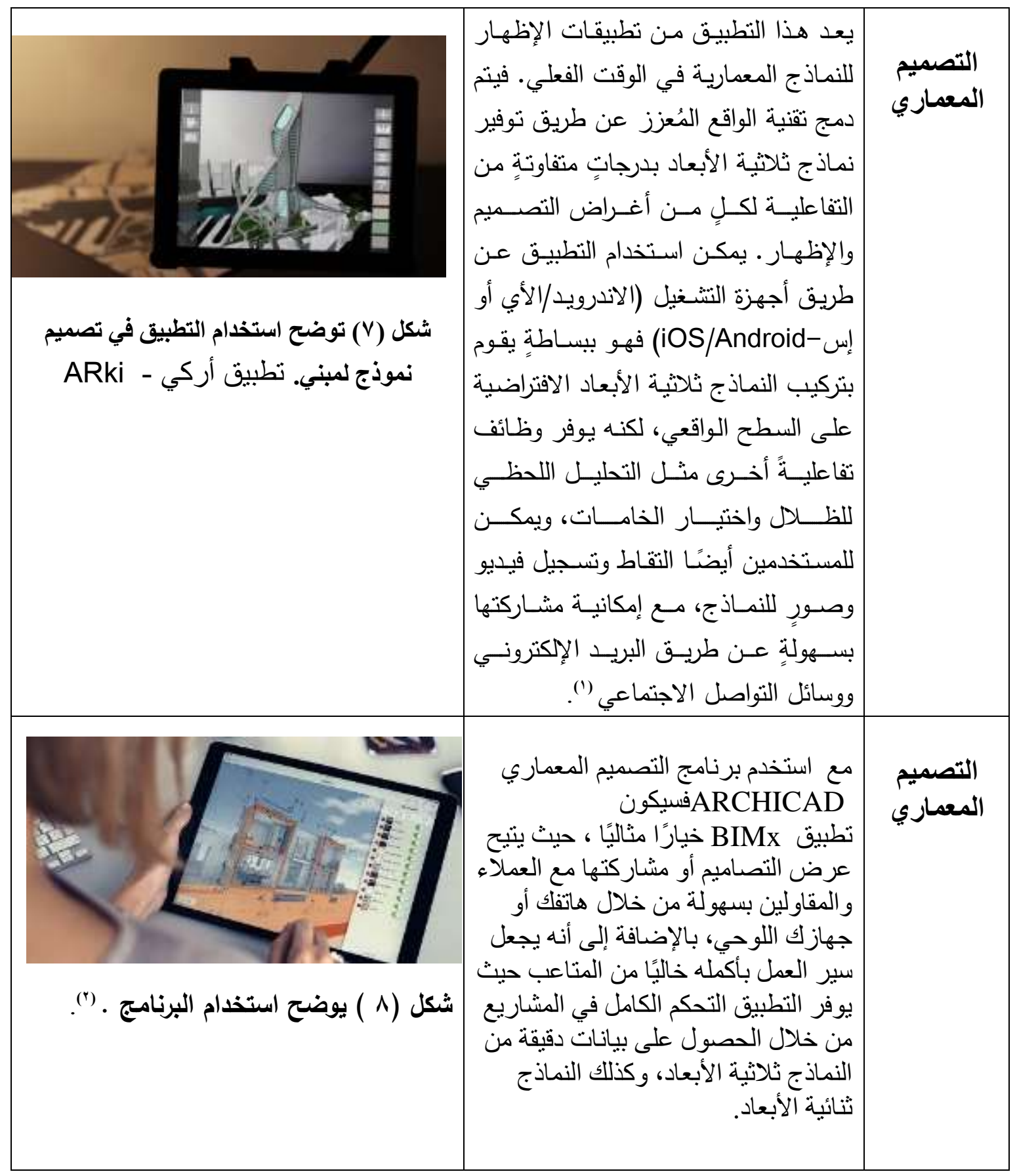

(1) The Top ${ }^{\circ}$ Virtual Reality and Augmented Reality Apps for Architects [Internet]. ArchDaily. $Y \cdot$ IV[cited $r \cdot \mid \wedge$ Oct $1 \cdot$ ]. Available

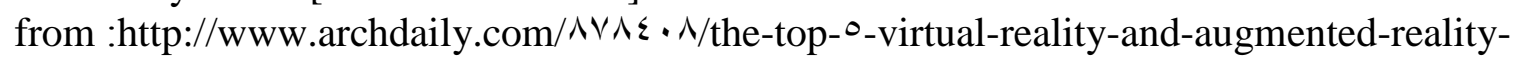
apps-for-architects

( ${ }^{\prime}$ ) https://aitnews.com/2019/03/13 


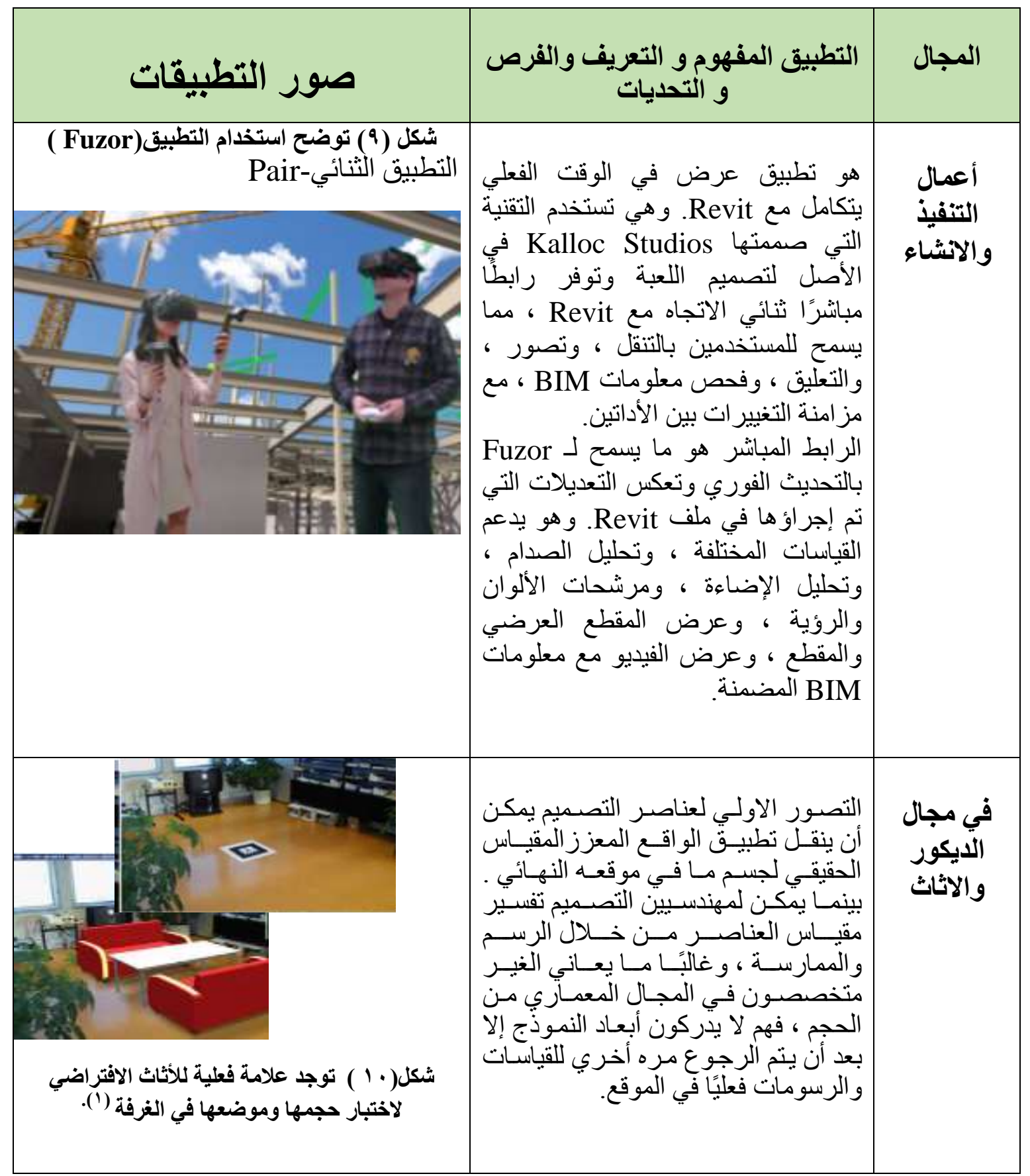

(1) Siltanen and Valtion Teknillinen Tutkimuskeskus (VTT), Theory and applications of marker-based augmented reality, Espoo 2012. VTT Science 3.198 p. + app. 43 p. 


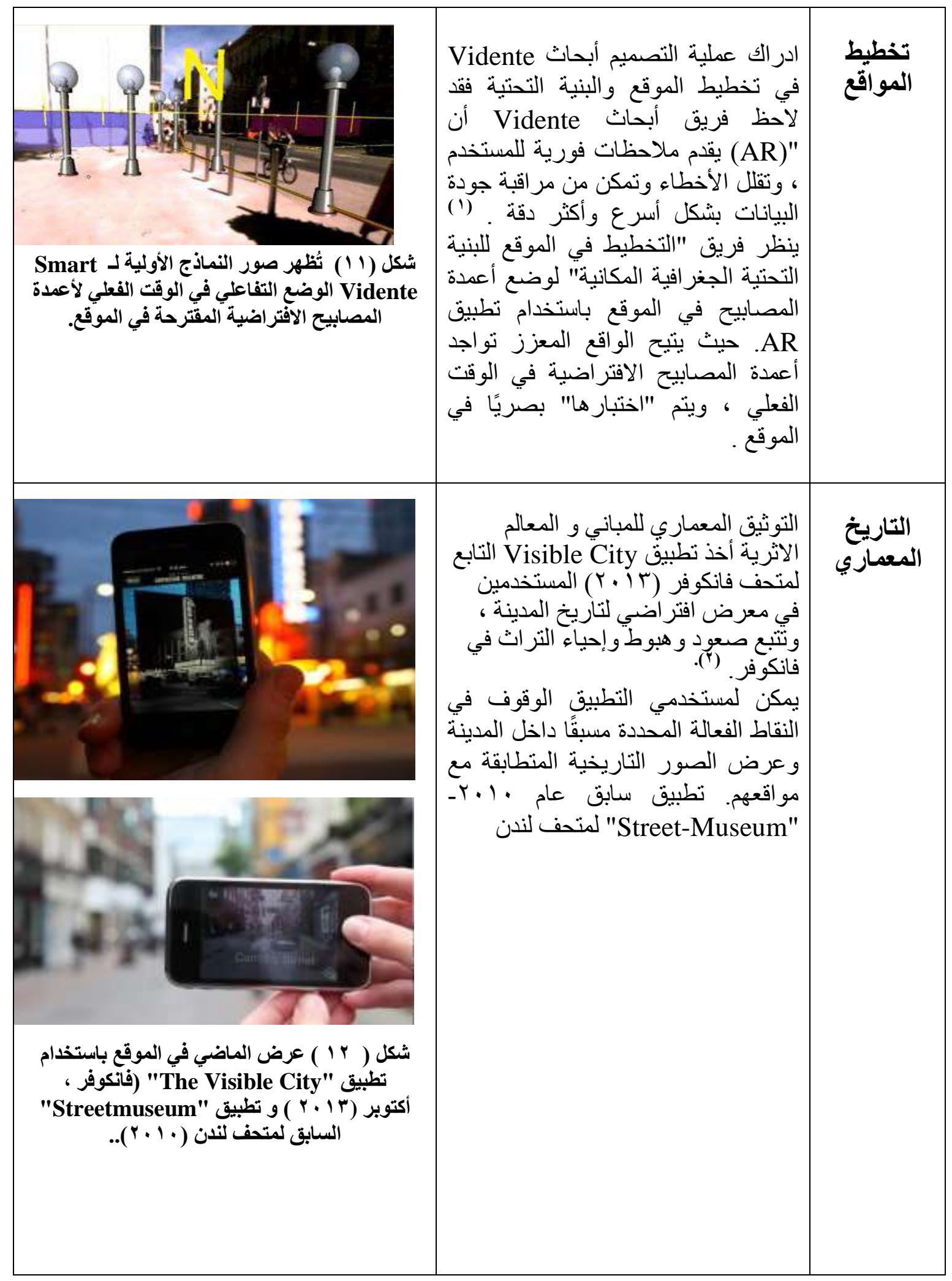

(1) Schall, Zollmann, and Reitmayr, "Bridging the Gap between Planning and Surveying with Augmented Reality User Interfaces."

(2) The Visible City. A Similar example was the Museum of London App, developed in (2010). . 


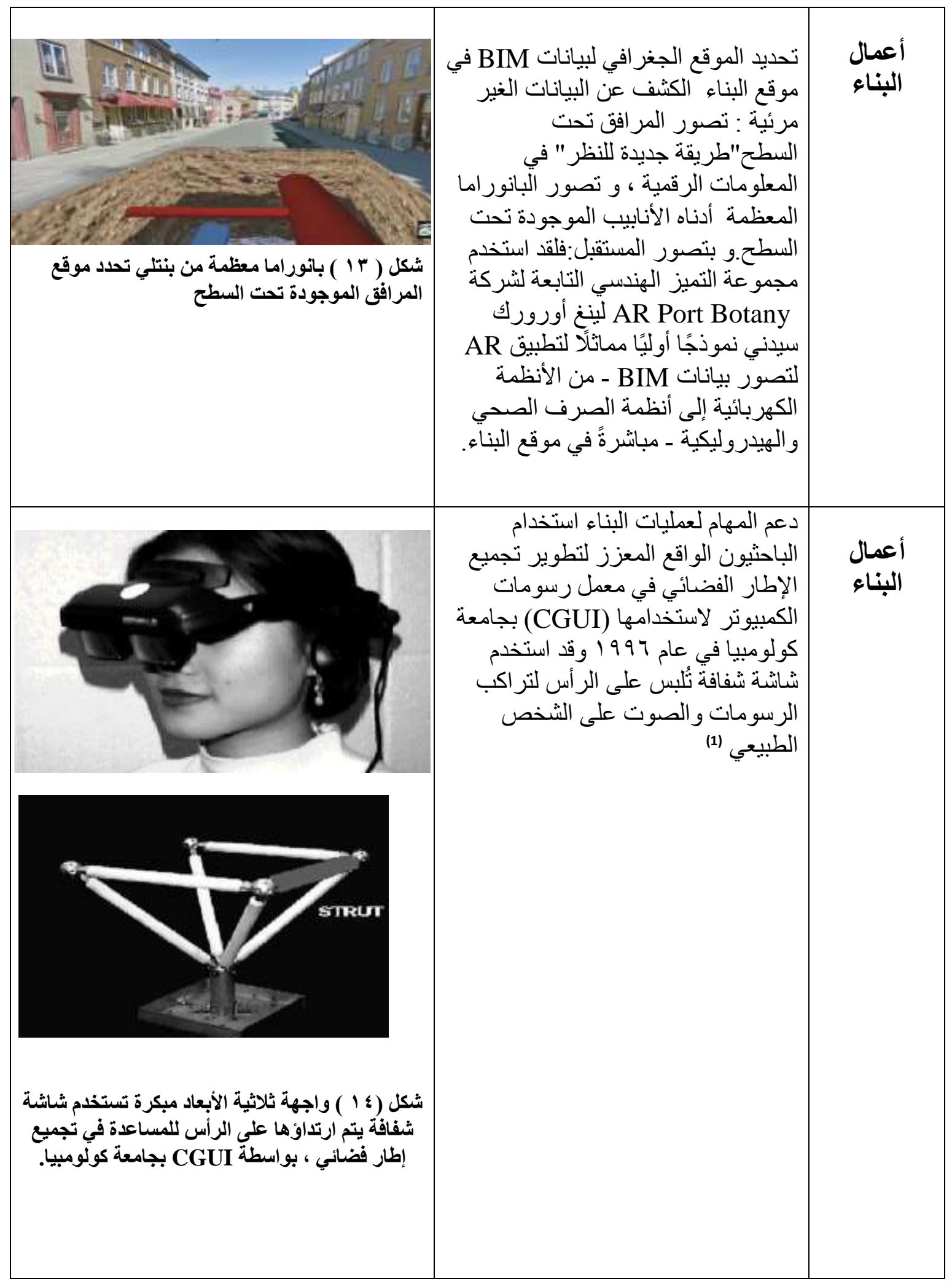

(1) Webster, Anthony \& Feiner, Steven \& Macintyre, Blair \& Massie, William \& Krueger, Theodore. (2000). Augmented Reality in Architectural Construction, Inspection, and Renovation. Proceedings of 1996 ASCE Congress on Computing in Civil Engineering. 


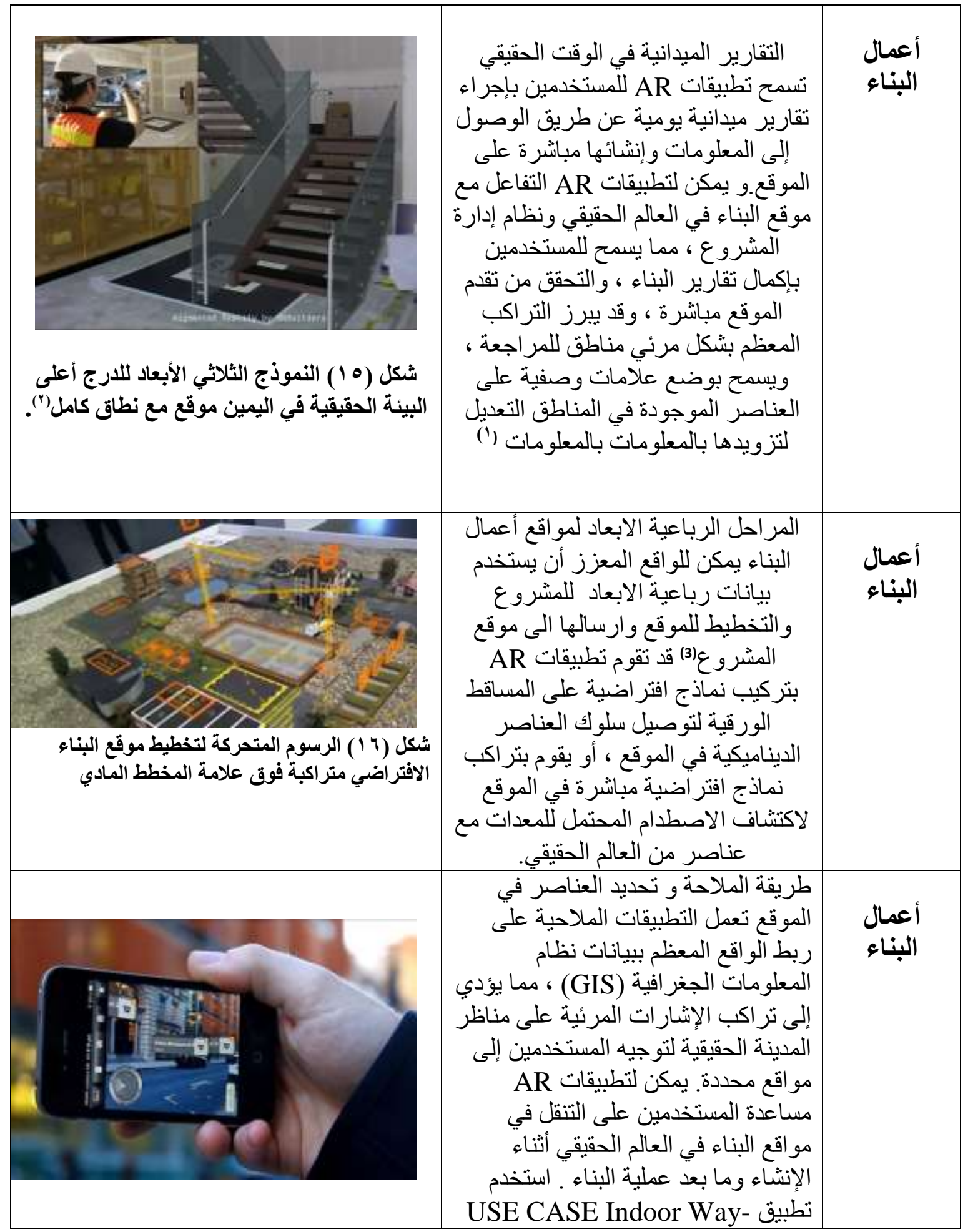

(1) This functionality is similar to Bentley's 'hypermodeling' feature, allowing markers floating within a virtual model to link to and call up a drawing representing that location. This improves the effectiveness of the information environment,

( ${ }^{\prime}$ ) http://www.perkinswill.com/work/institute-for-systemsbiology.html, 2013.

(3) "Barista, David. "Augmented reality goes mainstream: 12 applications for design and construction firms." N .p., n.d. Web. 4 September 2013. 


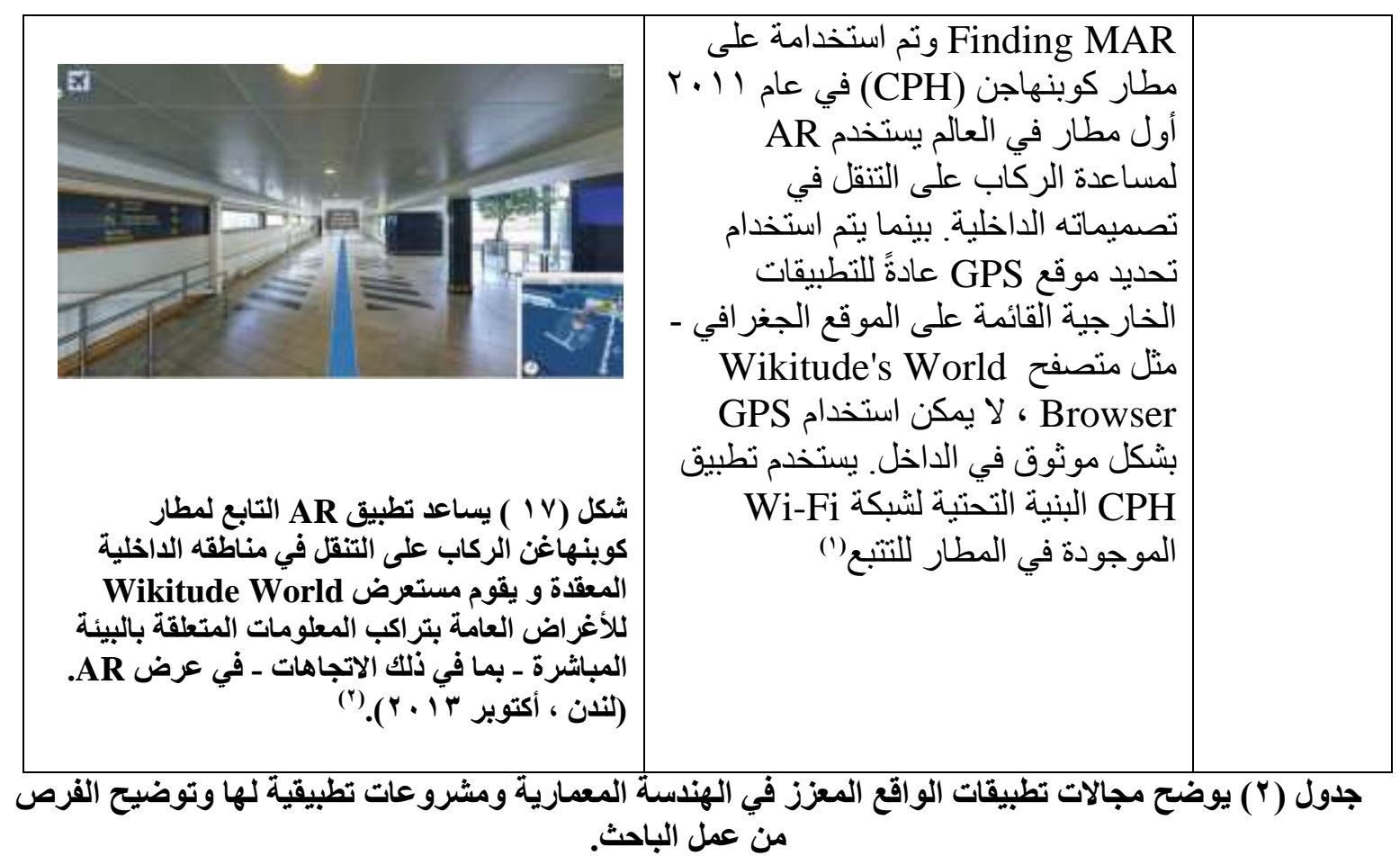

\section{اذن مما سبق نوضح فرص الواقع المعزز في الهندسة المعمارية : تصور كامل للتصميم في الموقع.

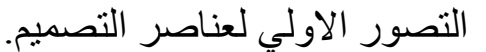

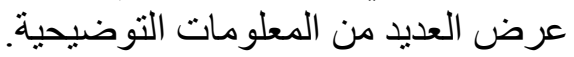 ادر الك عملية التصميم.

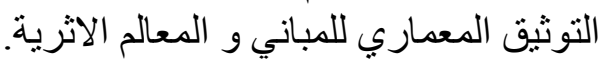

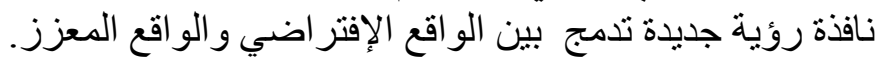

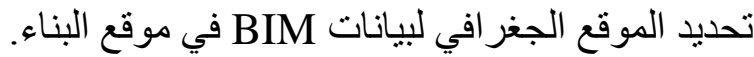 دعم المهام لعمليات البناء. التقارير الميدانية في الوقت الحقاء الحقيقي.

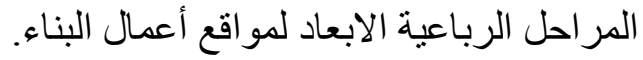 طريقة الملاحة و تحديد العناصر في الموقع.}

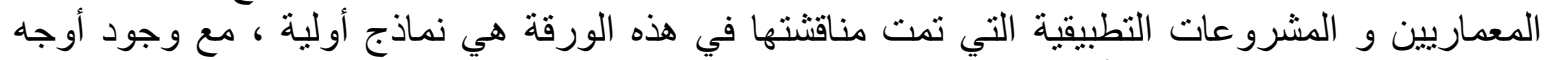
قصور تمنع حاليًا اعتمادها في أعمال المهندسين المعات المعارينين.

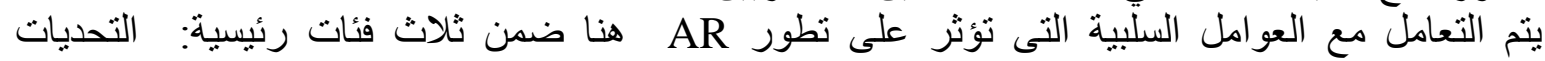
التكنولوجية و العو امل البشرية و القيود المالية.

(1) “Copenhagen Airport Unveils First 360 Degree Wayfinding App.” APR 2013

(2) "SITA and Copenhagen Airport Launch the World's First Indoor Augmented Reality Application | SITA.aero." 
أولا : التحديات التكنولوجية:

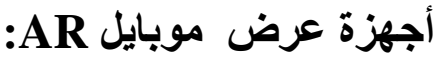

تستخدم معظم منصات AR اليوم أجهزة قائمة على الثاثنة (بما في ذللك الأجهزة اللوحية والهواتف

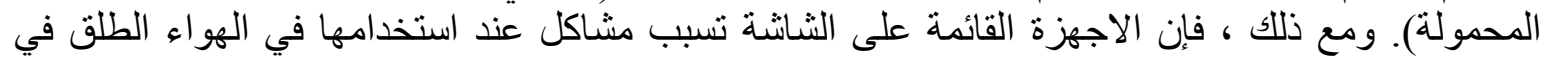

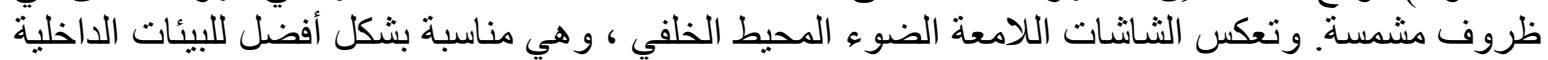

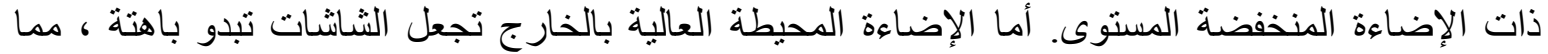

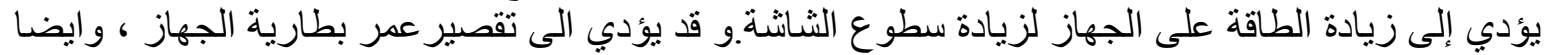

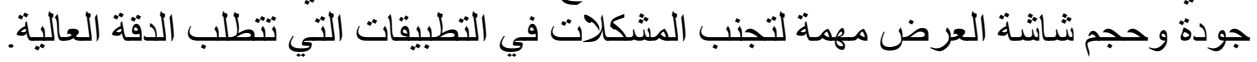

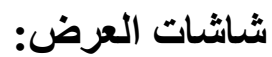

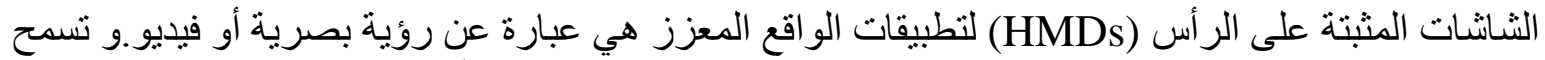

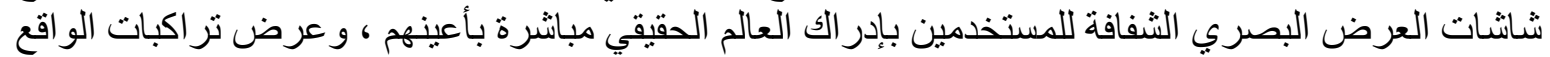

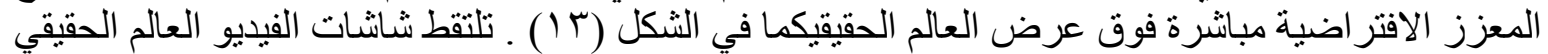

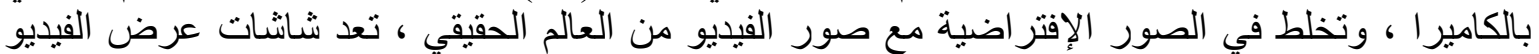

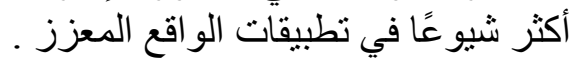
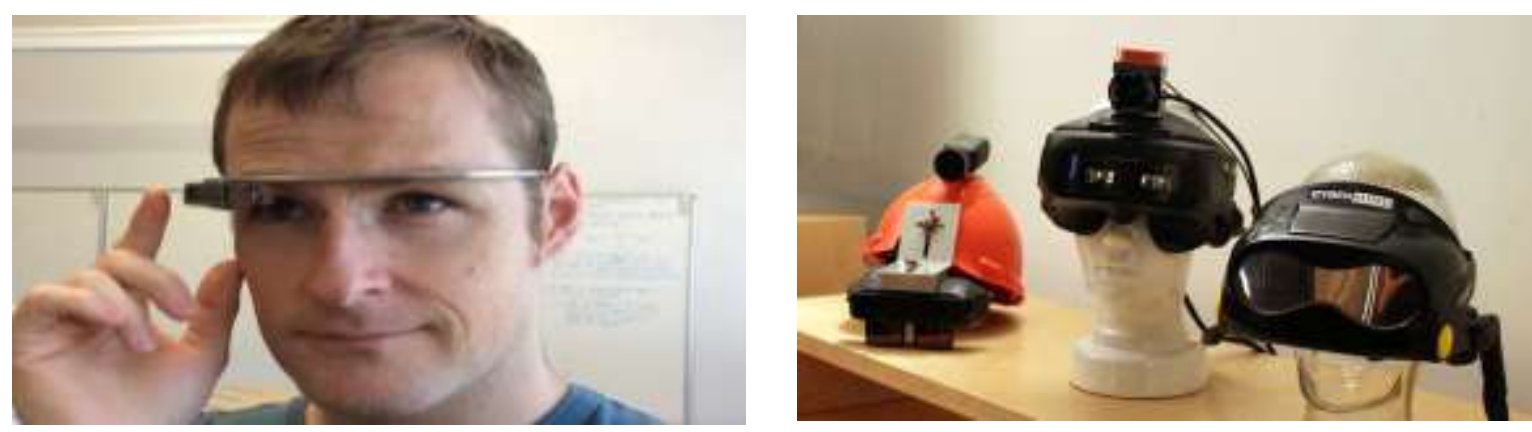

شكل ( ب r ) النموذج الأولي لشركة HMD (على اليمين) ، ونظارة Google Glass (على اليسار).

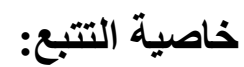

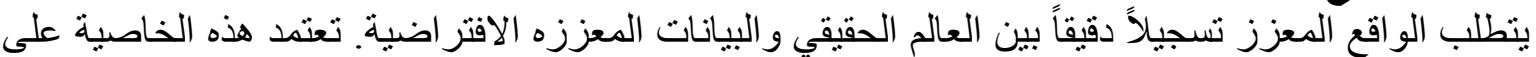

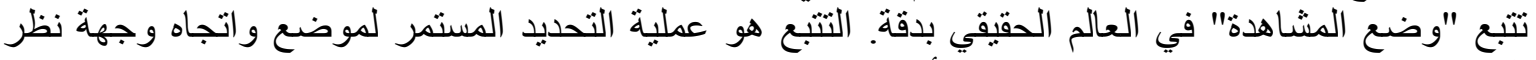

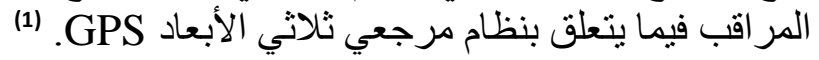

\section{عدم وجود عمليات قياسية صناعية :}

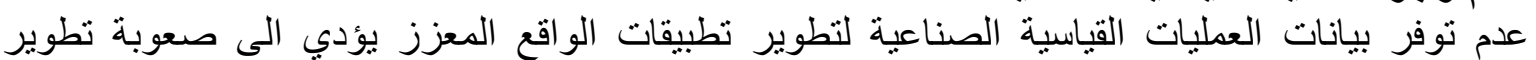

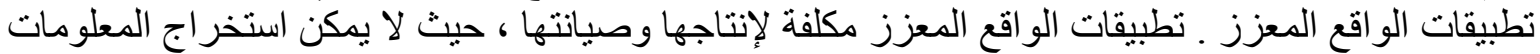

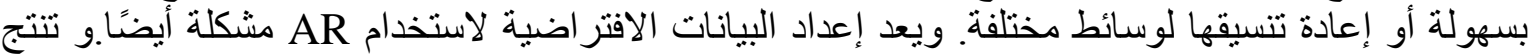

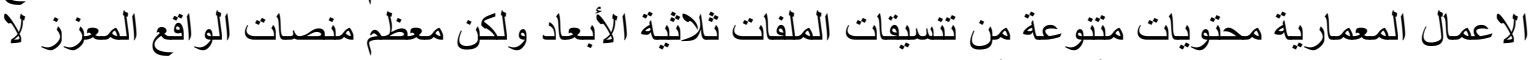
تدعم تنسيقات الملفات ثلاثية الأبعاد الأصلية.

(1)Perey and Terenzi, “Augmented Reality-Assisted 3D Visualisation for Urban Professional Users", April,2014 


\section{ثانيا: العامل البشري المؤثر على تكنولوجيا الواقع المعزز :} مقاومة التغيير: التئري

إن مطالبة الناس بالخروج من مناطق الراحة الخاصة بهم و استخدام التقنيات الجديدة سيواجه مقاومة أولية.

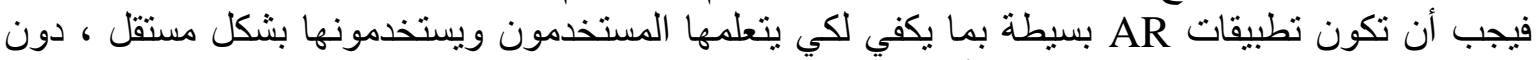

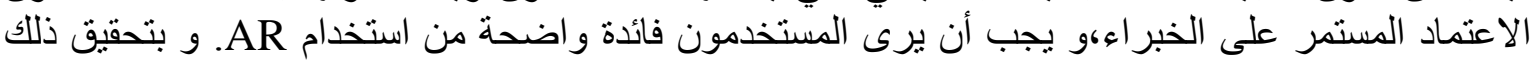
سوف يزداد الاقبال على تدريب AR و الاقتتاع به وعم استخدامة.

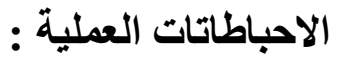

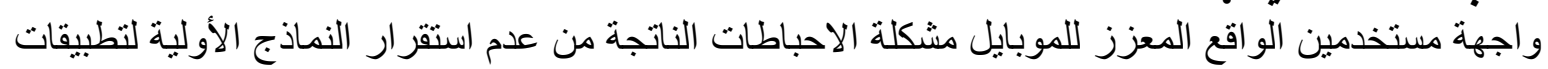
الو اقع المعزز بما يكفي للنطبيق التجاري.

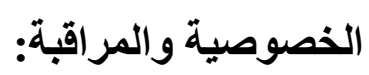

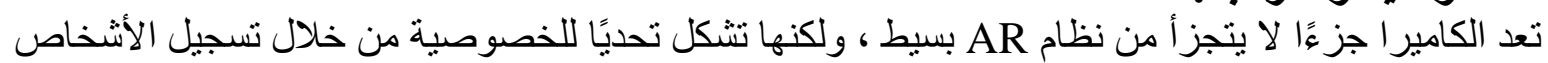

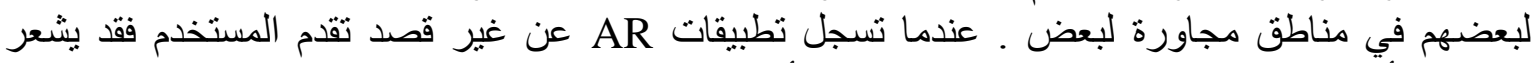
الاخرون أنهم تحت المر اقبة ، مما يؤدي إلى الإجهاد أو السلوك المتغير.

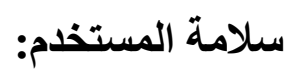

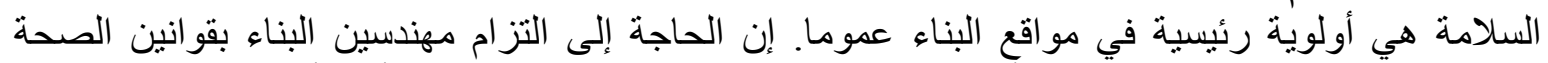

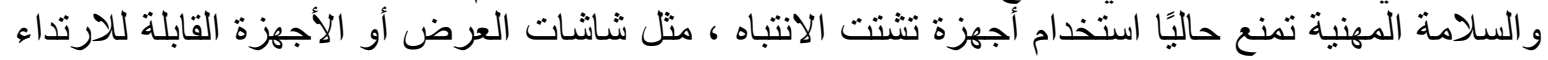

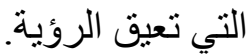

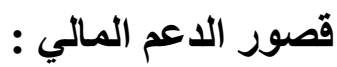

ستحتاج الثركات التعمارية التي تسعى إلى تطوير تطبيقات AR ودمجها في أعمالها إلى استثمار الوقت

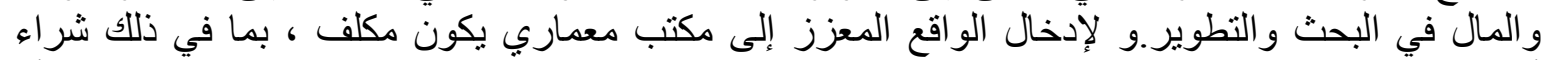

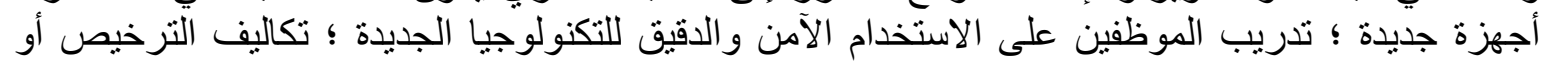

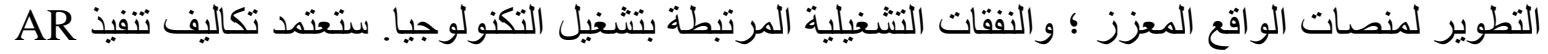
على الاستخدام المقصود منه ، وعلى العوائق التكنولوجية التي يجب التغلب التب عليها لتحقيق تجربة مستخدم

زاليادة الوعي وتغيير ثقافة العاملين بمجال الهندة المعمارية لديهم بأستعراض امكانيات الواقع

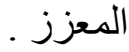
بسبب زياده الوعي لقد تم زيادة الطلب على تطبيقات الواقع المعزز مما يؤدي الى تطورة وتطور

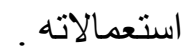

ه إمكانيات الو اقع المعزز للهندسة المعمارية لا حصر لها ، و الاستخدامات المقدمة لبست سوى البداية.

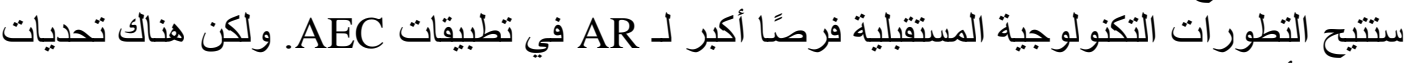

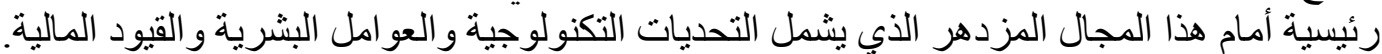
تطبيقات AR عالية المستوى عبر التصميم والبناء والتي تعد بتفاعل أكثر سهولة مع البيانات

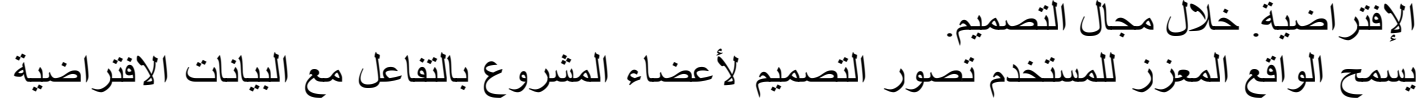
ونقل المظهر والحجم و الميزات المقصو دة للتصميم المقتر ح في سياقه النهائي. يساعد AR في تحجيم الدكونات و اكتثاف المشكلات. تقوم AR بتوضيح عملية التصميم نفسها لتمكين المصمين من تقدير سياق المشروع بشكل كامل. 
تقوم AR بتراكب بيانات BIM مباشرة على الموقع لتأكيد مواقع التثبيت لاعمال البناء و تحديد

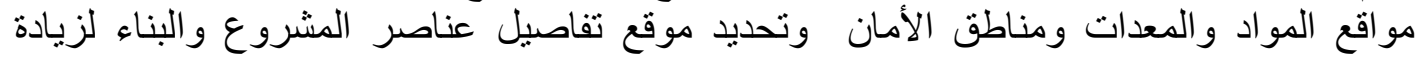
الكفاءة. تساعد AR عمال البناء في تتفيذ المهام المعقدة من خلال توفير تعليمات مسجلة ثلاثية الأبعاد مباثرة

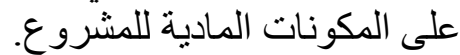

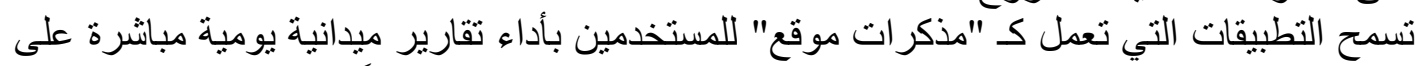

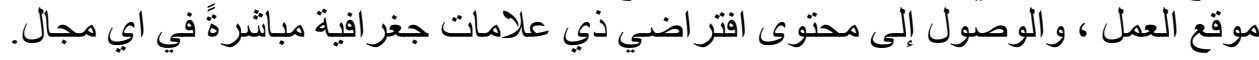

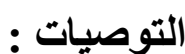
يجب أن تتغلب تطبيقات AR على التحديات التكنولوجية و الاجتماعية والمالية قبل اعتمادها في سير عمل المهندسين المعماريين. يجب تطوير شاشات و أجززة العرض المستقبلية إلى معالجة متطلبات السلامة الفريدة والظروف الخارجية لتطبيقات AR في البناء.

هجب تطوير أنظمة التتبع إلى تلبية المتطلبات المعقدة لاستخدامات البناء ، وتحقيق دقة عالية.

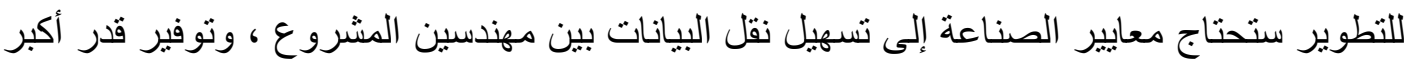
من قابلية التشغيل بين الوسائط المختلفة. مطلوب من المتخصصين المساعدة في تنفيذ مهام سير تنفيذ تطبيقات AR ولتوفير الوفير تدريب الموظفين

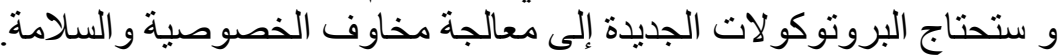
يجب على المنظمات التي تحرص على لئ تنفيذ AR في تدفقات عمل المشروع إلى الى القيام باستثمارات لابد من أن تعنتمد التكنولوجيا هيل على العديد المتخصصين في تطوير ها. ولكن مع المثابرة والوقت ،

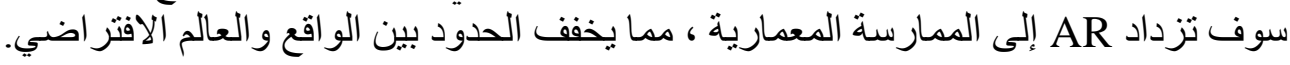

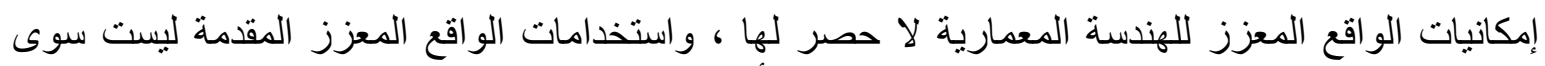

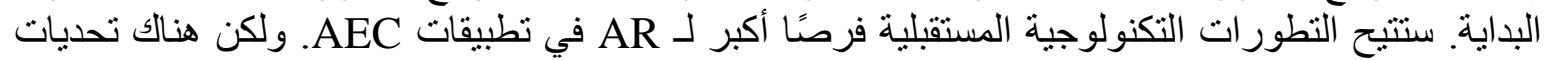

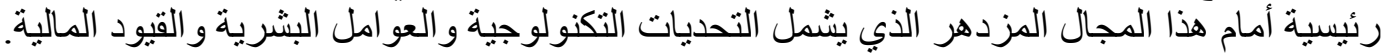
حددت الورقة تطبيقات AR عالية المستوى عبر التصميم والبناء و التي تعد بتفاعل أكثر سهولة مع البية البيانات

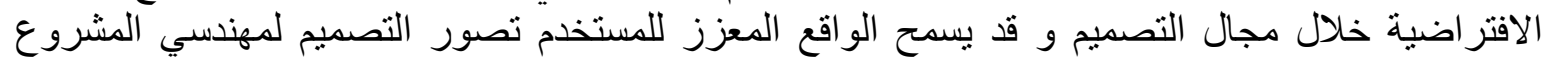

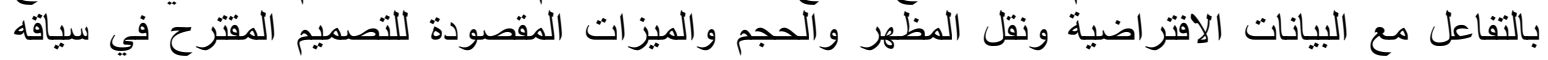
النهائي. قد يساعد AR في اكتشاف المشكلات ، وقائ وقد يزيد من وسائط العروض التقديمية المادية لتوصيل

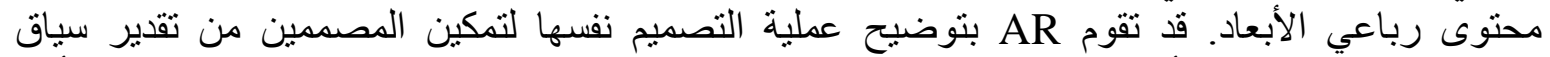

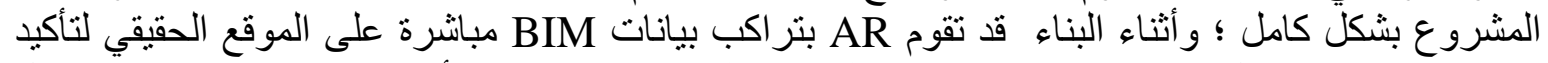

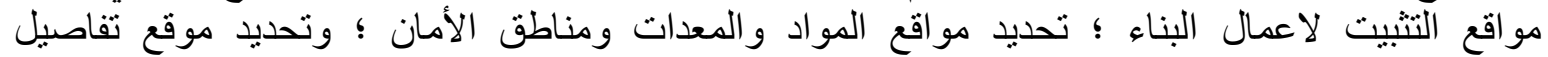

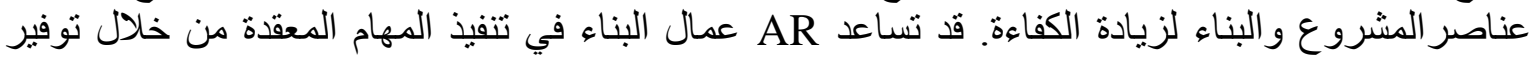

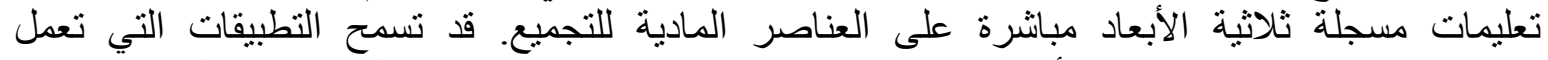

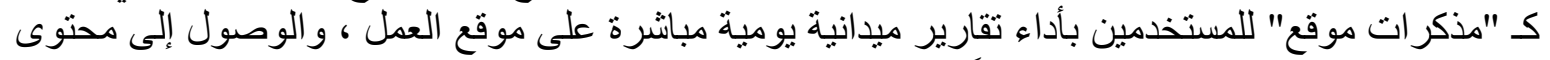

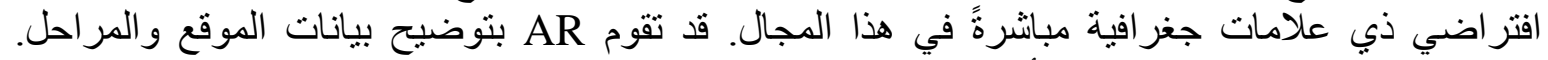
بالاقتران مع GIS ، قد يساعد AR أيضًا في التنقل في مواقع البناء.

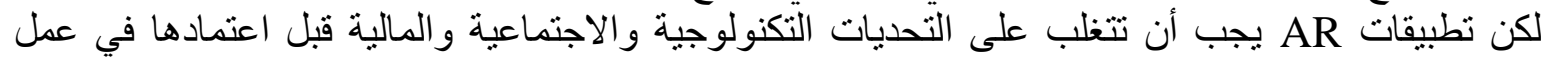

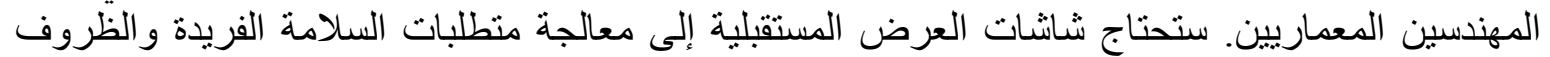

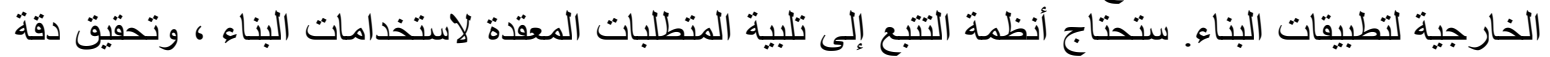

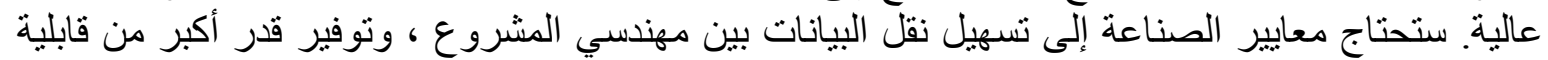

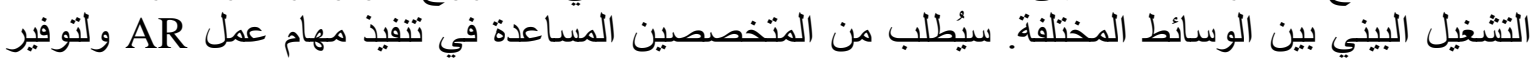




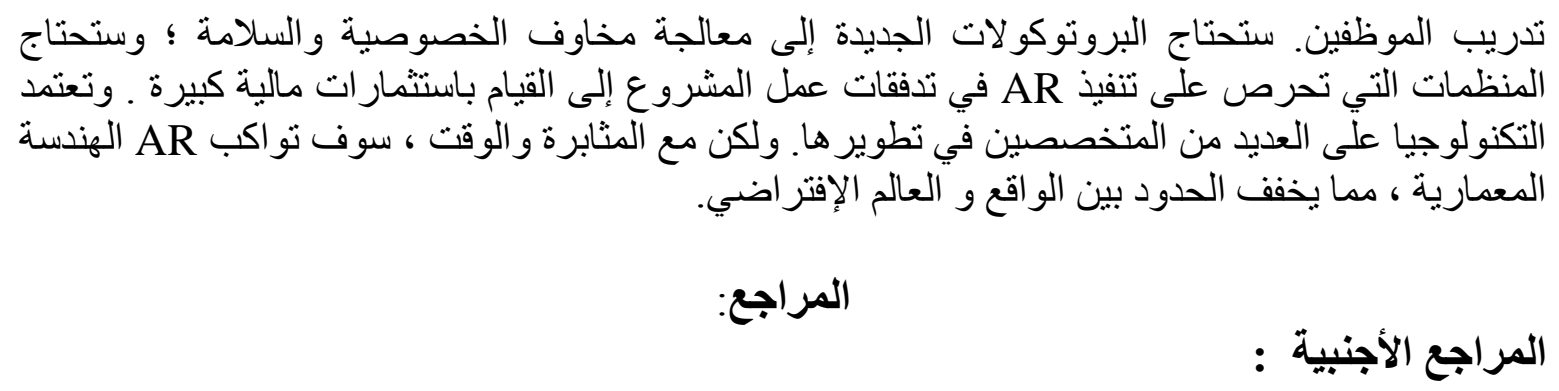

- Siltanen and Valtion Teknillinen Tutkimuskeskus (VTT), “Theory and applications of marker-based augmented reality", Espoo 2012. VTT Science 3.198 p. + app. 43 p.

- Olshannikova, Ekaterina \& Ometov, Aleksandr \& Koucheryavy, Yevgeni \& Olsson, Thomas. (2015)." Visualizing Big Data with augmented and virtual reality": challenges and research agenda. Journal of Big Data. 2. 10.1186/s40537015-0031-2.

- Schall, Gerhard ; Zollmann, Stefanie ; Reitmayr, Gerhard. "Bridging the gap between Planning and Surveying with Augmented Reality User Interfaces". Proceedings of Mobile HCI 2011. Pages r. r ro.

- Abboud, Rana, "Architecture in an Age of Augmented Reality: Opportunities and Obstacles for Mobile AR in Design, Construction, and Post-Completion”, NAWIC, . Pages r.-ro. March 2014

- Sugar \& Holmes, "Royal Australian Institute of Architects and Practice Services, You and Your Architect". Practice Services resource, Pages 1 - - - • •. April 1999.

- Webster, Anthony \& Feiner, Steven \& Macintyre, Blair \& Massie, William \& Krueger, Theodore. "Augmented Reality in Architectural Construction, Inspection, and Renovation”. Proceedings of 1996 ASCE Congress on Computing in Civil Engineering. 2000. Pages 00- 7 .

- Barista, David. "Augmented reality goes mainstream: 12 applications for design and construction firms." N.p, n.d. Web. 4 September 2013. . Pages $\vee V_{-} \vee \vee 0$.

- Perey and Terenzi, "Augmented Reality-Assisted 3D Visualisation for Urban Professional Users", April,2014 . Pages VY-V0.

- Wang, Xiangyu. "Using Augmented Reality to Plan Virtual Construction Worksite." International Journal of Advanced Robotic Systems 4, no. 4 (2007) . Pages 91-9r.

- Webster, Anthony, Steven Feiner, Blair MacIntyre, William Massie, and Theodore Krueger. "Augmented Reality in Architectural Construction, Inspection and Renovation." In Proc. ASCE Third Congress on Computing in Civil Engineering, 913-919, 1996 . Pages 1r-ı.

- Wilcox, David, Marvin Johnson, and Peter Carrato. "Augmented Reality: Bringing BIM to Life." Journal of Building Information Modeling no. Fall 2012 . Pages 11 19. 


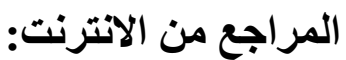

- http://curiosity.discovery.com/question/augmented-virtual-reality AccessDate:15/9/2020 2:30pm

- http://computer.howstuffworks.com/augmented-reality.htm AccessDate:20/9/2020 $\underline{5: 30 \mathrm{pm} \bullet}$

- http://www.t-immersion.com/augmented-reality/futurevision AccessDate:22/9/2020 7:20pm

- http://www.pocket-lint.com/news/108891-augmented-reality-travel-tourism-apps, 2013 AccessDate:29/9/2020 8:00 pm.

- https://www.futuretravelexperience.com/2011/03/copenhagen-embraces-mobiletechnology-with-augmented-reality-app/AccessDate:1·/^/2020 7:20pm

- https://www.youtube.com/watch?v=uqYAzklhORE AccessDate: 1 0/9/2020 7:20pm

- http://www.youtube.com/watch?v=uqYAzklhORE\&feature=youtube_gdata_player AccessDate: 1)/9/2020 7:1 Y pm

- The Visible City: Virtual Exhibition \& Mobile App, 2013 AccessDate: I $\_/ \wedge / 2020$ 9:20pm . http://www.youtube.com/watch?v=HyYgSBd_n5g\&feature=youtube_gdata_player AccessDate: $r \cdot / 9 / 2020 \leqslant: \cdot r \mathrm{pm}$ 